\title{
ENDOMORPHISM RINGS OF REDUCED TORSION-FREE MODULES OVER COMPLETE DISCRETE VALUATION RINGS
}

BY

\author{
WOLFGANG LIEBERT ${ }^{1}$ )
}

\begin{abstract}
The purpose of this paper is to find necessary and sufficient conditions that an abstract ring be isomorphic to the ring of all endomorphisms of a reduced torsion-free module over a (possibly noncommutative) complete discrete valuation ring.
\end{abstract}

Introduction. Let $R$ be a complete discrete valuation ring and $H$ a reduced torsion-free $R$-module. The structure of the $R$-endomorphism ring $E_{R}(H)$ of $H$ is to a large extent determined by the ideal $E_{0}(H)$ of all those $R$-endomorphisms of $H$ which map $H$ onto a submodule of finite rank. If $R$ is a (possibly noncommutative) division ring, then it is well known that $E_{0}(H)$ is the right socle (sum of all minimal right ideals) as well as the left socle of $E_{R}(H)$. However, if $R$ is not a division ring, then $E_{R}(H)$, just like $R$, does not possess minimal ideals at all. In this general case it turns out that $E_{0}(H)$ is both the sum of all minimal nonradical right ideals and the sum of all minimal nonradical left ideals of $E_{R}(H)$. By a minimal nonradical right (left) ideal of a ring $E$ we mean a right (left) ideal $I$ which is not contained in the Jacobson radical $J(E)$ of $E$ and has the property that every right (left) ideal of $E$ which is properly contained in $I$ belongs to $J(E)$.

In $\$ 2$ we briefly comment on the Jacobson radical of $E_{R}(H)$. In $\$ 3$ we investigate $E_{0}(H)$ and characterize the subrings of $E_{R}(H)$ which contain $E_{0}(H)$. In $\$ 4$ we show that a subring $S$ of $E_{R}(H)$ which contains $E_{0}(H)$ is complete in its finite topology if and only if $S=E_{R}(H)$. This topology on $E_{R}(H)$ is defined by taking the annihilators of the finite subsets of $H$ as neighborhoods of zero.

Finally, $\$ 5$ is devoted to a characterization of $E_{R}(H)$ for reduced torsionfree $R$-modules $H$ which are complete in their $p$-adic topology. The endomorphism rings of these modules are complete in their J-finite topology which is obtained by taking as neighborhoods of zero the right ideals in $E_{R}(H)$ which map finite subsets of $H$ into $p^{i} H$. We note that our characterization of $E_{R}(H)$ for complete

Presented to the Society, January 23, 1970; received by the editors February 24, 1970. AMS 1969 subject classifications. Primary 1650; Secondary 2030, 1698.

Key words and phrases. Complete discrete valuation ring, reduced torsion-free module, end omorphism ring, finite endomorphis ms, Jacobson radical, sum of all minimal nonradical right (left) ideals, finite topology and $J$-finite topology on endomorphism rings.

(1) This research was sponsored by NSF Grant No. GP-13286. 
$H$ in [4] did not involve these two topologies. However, the methods developed there are not applicable to the case where $H$ is not complete. Our methods here are somewhat analogous to those used in [3] where we characterized the endomorphism rings of abelian p-groups without elements of infinite height.

1. Definitions and preliminaries. An ideal of $E$ is called (non)radical if it is (not) contained in $J(E)$. We define the $J$-adic topology of $E$ by taking the powers of $J(E)$ as a neighborhood basis at zero.

Definition. A ring $R$ is a complete discrete valuation ring if it satisfies the following four conditions:

(1) $R / J(R)$ is a division ring.

(2) $J(R)=p R=R p$, where $p$ is either zero or a nonnilpotent element of $R$.

(3) $R$ is Hausdorff and complete in its J-adic topology.

Then $R$ possesses an identity element which is obtained by lifting the identity element from $R / J(R)$.

Some of the elementary properties of $R$ have been listed in [4]. If $p=0$ in the definition above, then $R$ is a division ring and the $R$-modules are just the vector spaces. Since their endomorphism rings have already been characterized by Wolfson [7], we shall henceforth assume that $p \neq 0$.

Throughout this paper $R$ will always be a complete discrete valuation ring with prime $p$ as defined above. The term $R$-module should be interpreted as meaning a unitary left $R$-module. Let $H$ be an $R$-module. Then $H$ is called reduced if it has no nonzero divisible submodules. If an element $y$ in $H$ is divisible by $p^{n}$ but not by $p^{n+1}$, then we say that $y$ has height $n$ and write $h(y)=n$; it has infinite height if it is divisible by $p^{n}$ for every $n$. A submodule $B$ of $H$ is pure if $p^{n} B=B \cap p^{n} H$. In a torsion-free $R$-module, the elements of infinite height form a divisible submodule. Consequently, if $H$ is reduced and torsion-free, then it is without elements of infinite height: $\bigcap_{n=1}^{\infty} p^{n} H=0$. We introduce the $p$-adic topology on such a module by using the submodules $p^{n} H$ as neighborhoods of 0 . If $H$ is complete in its $p$-adic topology, we shall simply call it complete. We define the rank of $H$ to be the cardinal number of any of its maximal linearly independent subsets. If $S$ is a submodule of $H$, then the corank of $S$ is the rank of $H / S$. If $T$ is a subset of $H$, then $\{T\}$ denotes the submodule generated by $T$.

The reader must keep in mind that we do not require commutativity of $R$ throughout this paper. We are interested here in reduced torsion-free $R$-modules, and we assert that their theory is identical with the theory of the corresponding modules over commutative valuation rings as presented in Kaplansky [2]. We do not repeat proofs of the following well-known simple facts about these modules which we shall use frequently. 
(1.1) Every reduced nonzero torsion-free $R$-module $H$ has a nonzero cyclic direct summand. In fact, every element $x$ of $H$ which is not in $p H$ generates a direct summand [2, Corollary 1, p. 53].

(1.2) A reduced torsion-free $R$-module of countable rank is free [2, Theorem 20, p. 48].

(1.3) Let $M$ be any $R$-module. Then any pure submodule of finite rank is a direct summand of $M[2$, Exercise 57, p. 54].

(1.4) $R$ is the only reduced torsion-free indecomposable $R$-module [2, Corollary 2, p. 53].

(1.5) Every finite subset, and hence every submodule of finite rank, of a reduced torsion-free $R$-module $H$ can be embedded into a direct summand of $H$ of finite rank.

Next, let $E$ be an arbitrary ring. If $T$ is any subset of $E$, then $\varrho_{E}(T)$ is the set of all $\sigma$ in $E$ such that $\sigma T=0$. The right annihilator $R_{E}(T)$ is defined similarly.

The elements of $E_{R}(H)$ shall operate on the elements of $H$ on the right. Now let $E$ denote any subring of $E_{R}(H)$. If $S$ is any subset of $H$, then $P_{E}(S)$ is the totality of elements $\zeta$ in $E$ such that $S \zeta=0$, and $\Lambda_{E}(S)$ is the totality of $a$ in $E$ for which $H \alpha \subseteq S$. If $S$ is actually a submodule, then $P_{E}(S)$ is a right ideal and $\Lambda_{E}(S)$ a left ideal of $E$. If $Q$ is any subset of $E$, then $K(Q)$ is the totality of $x$ in $H$ for which $x Q=0$, and $H Q$ is the set of elements $b \beta$ for $b \in H$ and $\beta \in Q$. Whenever $E=E_{R}(H)$, we shall write the operators $P, \Lambda, R, \mathcal{Q}$ without subscript.

We state the following useful generalization of results which are proven in [7]. The proofs go over unchanged to the present case.

(1.6) Lemma. Let $H$ be any $R$-module and $E$ any subring of $E_{R}(H)$. Then

(1) $\Lambda_{E}[K(T)]=\mathscr{L}_{E}(T)$ for every subset $T$ of $E$.

(2) $P_{E}(H T)=R_{E}(T)$ for every subset $T$ of $E$.

(3) $P_{E}(S)=R_{E}\left[\Lambda_{E}(S)\right]$ for every submodule $S$ of $H$ satisfying $H \Lambda_{E}(S)=S$.

(4) $\Lambda_{E}(S)=\mathscr{L}_{E}\left[P_{E}(S)\right]$ for every submodule $S$ of $H$ satisfying $K\left[P_{E}(S)\right]=S$.

2. The Jacobson radical. Let $H$ be a reduced torsion-free $R$-module. The problem of characterizing the Jacobson radical of $E_{R}(H)$ turns out to be extremely difficult. The following lemma contains all we are able to say about it for arbitrary $H$.

(2.1) Lemma. Let $E$ be a subring of $E_{R}(H)$ which contains $E_{0}(H)$. Then $J(E) \subseteq \Lambda_{E}(p H)$.

Proof. Suppose that $J(E) \nsubseteq \Lambda_{E}(p H)$. Then there exist $x \in H$ and $a \in J(E)$ such that $x \alpha \notin p H$. By (1.1), there is a $\zeta \in E_{0}(H)$ such that $(x \alpha) \zeta=x$. But $\alpha \zeta \in J(E)$. Hence $\alpha \zeta$ is quasi-regular. Therefore, we can find $\beta \in E$ with 
$\alpha \zeta+\beta-\alpha \zeta \beta=0$. Then

$$
x=x \alpha \zeta=x(\alpha \zeta \beta-\beta)=x a \zeta \beta-x \beta=x \beta-x \beta=0 .
$$

which is a contradiction.

(2.2) Corollary. $\bigcap_{n=1}^{\infty} J(E)^{n}=0$.

The following two examples show that for some modules $H$ the inclusion relation in (2.1) is equality, for others it is a proper inclusion.

(2.3) Theorem. Let $H$ be a reduced complete torsion-free $R$-module. Then there exists a non-zero-divisor $\pi$ in $E_{R}(H)$ sucb that $J\left[E_{R}(H)\right]=\Lambda(p H)=\pi E_{R}(H)$ $=E_{R}(H)_{\pi}$. Moreover, $E_{R}(H)$ is Hausdorff and complete in its J-adic topology [4, Theorem 5.6].

(2.4) Proposition. If $H$ is free of infinite rank, then $J\left[E_{R}(H)\right]$ is properly contained in $\Lambda(p H)$.

Proof. Let $H=G \oplus \sum_{k=1}^{\infty}\left\{b_{k}\right\}$, where the latter sum is direct. Let $\alpha \in E_{R}(H)$ be defined by $G \alpha=0$ and $b_{k} a=p b_{k+1}$ for all $k$. Clearly $\alpha \in \Lambda(p H)$. Assume, by way of contradiction, that $a \in J\left[E_{R}(H)\right]$. Then $1-\alpha$ is a unit of $E_{R}(H)$, that is to say, an $R$-automorphism of $H$. Therefore, there exists $x \in H$ such that $x(1-\alpha)=b_{1}$. Let $x=g+\sum_{k=1}^{n} r_{k} b_{k}$, where $g \in G$ and $r_{k} \in R$. Then

$$
\begin{aligned}
b_{1} & =g(1-\alpha)+\sum_{k=1}^{n} r_{k} b_{k}(1-\alpha)=g+\sum_{k=1}^{n}\left(r_{k} b_{k}-r_{k} p b_{k+1}\right) \\
& =g+r_{1} b_{1}+\left[\sum_{k=2}^{n}\left(r_{k}-r_{k-1} p\right) b_{k}\right]-r_{n} p b_{n+1}
\end{aligned}
$$

implies that $g=0, r_{n} p=0$ and $r_{k}-r_{k-1} p=0$ for $k=2, \cdots, n$. Since $p$ is not a zero-divisor in $R$ [4, Property 2.4], we conclude that $r_{k}=0$ for $k=1, \cdots, n$. Consequently, $b_{1}=0$, which is a contradiction.

The fact that without the completeness hypotheses we can say virtually nothing about $J\left[E_{R}(H)\right]$ is entirely analogous to the situation for the endomorphism rings of $p$-groups without elements of infinite height (see [5]). In the next section we will, however, determine $J\left[E_{0}(H)\right]$.

3. The finite endomorphisms. Throughout this paper, $H$ denotes an arbitrary reduced torsion-free $R$-module. An $R$-endomorphism $\alpha$ of $H$ is called finite if the submodule $H a$ of $H$ has finite rank. It is immediate that $E_{0}(H)$, the set of all finite $R$-endomorphisms of $H$, is a two-sided ideal of $E_{R}(H)$. The following lemma shows that there are plenty of elements in $E_{0}(H)$. 
(3.1) Lemma. If $x, y$ are arbitrary elements of $H$, then there exists a finite $R$-endomorphism of $H$ mapping $x$ onto $y$ if and only if $b(x) \leq b(y)$.

Proof. Exercise 72 of [2].

Our main objective in this section is to characterize $E_{0}(H)$ in purely ringtheoretical terms. The key lemma for this characterization is the following one.

(3.2) Lemma. Let a be a finite R-endomorphism of $H$. Then $K(\alpha)$ is a direct summand of $H$. Moreover, there exists a direct decomposition $H=F \oplus K$ of $H$ sucb that $F$ bas finite rank, $H \alpha \subseteq F$ and $K \subseteq K(\alpha)$.

Proof. Since $H \alpha$ has finite rank, it is free, by (1.2). Any epimorphism $H \rightarrow$ $M$, where $M$ is free, is direct. Therefore, the kernel $K(\alpha)$ of $\alpha$ is a direct summand of $H$. Hence, we can write $H=G \oplus K(a)$ with $G$ of finite rank. Then $G+$ $H \alpha=G+G \alpha$ has finite rank. Denote by $S$ the intersection of $G+H \alpha$ and $K(\alpha)$. By (1.5) there exist submodules $N$ and $K$ of $K(\alpha)$ such that $K(\alpha)=N \oplus K$ and $N$ has finite rank and $S \subseteq N$. Then $H$ is the direct sum of $F=G+N$ and $K$ with $F$ of finite rank, $H \alpha \subseteq F$ and $K \subseteq K(\alpha)$.

An indempotent $e$ of a ring $E$ is called minimal if $e \neq f+g$, where $f$ and $g$ are idempotents in $E$ with $f g=0=g f$. Using (1.4), we obtain

(3.3) Lemma. Let $E$ be a subring of $E_{R}(H)$ which contains $E_{0}(H)$. The following propetties of $\sigma \in E$ are equivalent:

(1) $\sigma$ is a minimal idempotent of $E$,

(2) $H \sigma$ is an indecomposable direct summand of $H$,

(3) $\mathrm{Ho}$ is a direct summand of rank 1.

The following result is an immediate consequence of (1.2), (3.2) and (3.3).

(3.4) Corollary. Let $E$ be a subring of $E_{R}(H)$ which contains $E_{0}(H)$. Then $E_{0}(H)=\sum_{\pi \in \Phi(E)} \pi E=\sum_{\pi \in \Phi(E)} E \pi$, where $\Phi(E)$ is the set of all minimal idempotents of $E$.

We need two further lemmas before we can characterize $E_{0}(H)$. We call a right (left) ideal $I$ of a ring $E$ minimal nonradical if $I$ is not contained in $J(E)$ and if every right (left) ideal $Y$ of $E$, which is properly contained in $I$, is contained in $J(E)$.

(3.5) Lemma. Let $E$ be a ring and e a minimal idempotent of $E$. Then the following are equivalent:

(1) $e E$ is a minimal nonradical right ideal of $E$,

(2) $e E e / J(e E e)$ is a division ring.

(3) Ee is a minimal nonradical left ideal of E [4, Lemma 9.1]. 
(3.6) Lemma. Let $E$ be a subring of $E(H)$ which contains $E_{0}(H)$. Then the following three properties of a left [right] ideal $X$ of $E$ are equivalent:

(1) There exists a direct summand $F$ of rank [corank] one in $H$ such that $X=\Lambda_{E}(F)\left[X=P_{E}(F)\right]$.

(2) $X$ is a principal left [right] ideal generated by a minimal idempotent.

(3) $X$ is a minimal nonradical left $[$ right] ideal.

Proof. The equivalence of (1) and (2) follows from (3.3).

Assume that (2) is true, that is to say, $X=E \sigma[X=\sigma E]$ with $\sigma$ a minimal idempotent. Then, applying (3.3), $\sigma E \sigma=\sigma E_{R}(H)_{\sigma} \cong E_{R}(H \sigma) \cong R$. But $R / J(R)$ is a division ring. Hence the validity of (3) is a consequence of (3.5).

Next, let $X$ be a minimal nonradical right ideal of $E$. Then $X^{2}=X$, because otherwise $X^{2} \subseteq J(E)$ would imply $X \subseteq J(E)$. Now $X \subseteq \Lambda_{E}(p H)$ would imply $X=$ $X^{n} \subseteq \Lambda_{E}\left(p^{n} H\right)$ for every $n \geq 1$, hence $X \subseteq \bigcap_{n=1}^{\infty} \Lambda_{E}\left(p^{n} H\right)=\Lambda_{E}\left(\bigcap_{n=1}^{\infty} p^{n} H\right)=$ $\Lambda_{E}(0)=0$, which is impossible. Therefore, $X \nsubseteq \Lambda_{E}(p H)$. So we can find $x \in H$ and $\alpha \in X$ such that $x \alpha \notin p H$. Hence $x \notin p H$, since $p H$ is fully invariant. By (1.1) we can write $H=\{x \alpha\} \oplus G$. Let $\zeta \in E$ be defined by $(x \alpha) \zeta=x$ and $G \zeta=0$. Then $H a \zeta=\{x\}$. Hence, by (3.2), $H=\{y\} \oplus K(a \zeta)$ for some $y \in H$. Now, by (2.1), $\alpha \zeta \notin J(E)$, since $x a \zeta=x \notin p H$. Also $\alpha \zeta \in P_{E}[K(\alpha \zeta)] \cap X$. Therefore, the minimality of $X$ implies $X \subseteq P_{E}[K(\alpha \zeta)]$. But since the implication (1) $\rightarrow(3)$ has already been established, $P_{E}[K(\alpha \zeta)]$ is minimal nonradical. Hence, $X=$ $P_{E}[K(\alpha \zeta)]$.

Finally, let $L$ be a minimal nonradical left ideal. Then, by the same argument as before in the right ideal case, there must exist $y \in H$ and $\lambda \in L$ with $y \lambda \notin p H$. Hence $y \notin p H$, and by (1.1) we can write $H=\{y\} \oplus M$. Define $\sigma \in E$ by $y \sigma=y$ and $M \sigma=0$. Then, in view of (2.1), $y \sigma \lambda=y \lambda \notin p H$ shows that $\sigma \lambda \notin$ $J(E)$. But $\sigma \lambda \in L \cap \Lambda_{E}(\{y \lambda\})$. Therefore, the minimality of $L$ implies $L=$ $\Lambda_{E}(\{y \lambda\})$. But $\{y \lambda\}$ is a direct summand of $H$, by (1.1), and we have already shown that $\Lambda_{E}(\{y \lambda\})$ is a minimal nonradical left ideal. Hence, $L=\Lambda_{E}(\{y \lambda\})$. Thus, (1) is a consequence of (3), and the lemma is proved.

By combining (3.4) and (3.6) we arrive at the following ideal-theoretic characterization of $E_{0}(H)$.

(3.7) Theorem. Let $E$ be a subring of $E_{R}(H)$ which contains $E_{0}(H)$. Then $E_{0}(H)$ is both the sum of all minimal nonradical right ideals and the sum of all minimal nonradical left ideals of $E$.

The fact that $E_{0}(H)$ is a two-sided ideal of $E_{R}(H)$ has the following consequence.

(3.8) Proposition. Let $E$ be a subring of $E_{R}(H)$ which contains $E_{0}(H)$. Then every right (left) ideal in the ring $E_{0}(H)$ is a right (left) ideal in the ring $E$. 
Proof. Let $X$ be a right (left) ideal of $E_{0}(H)$ and let $\zeta \in E$. We have to show that $X \zeta \subseteq X(\zeta X \subseteq X)$. Let $\alpha \in X$. Then it follows from (3.2) that there exists an idempotent $\sigma$ in $E_{0}(H)$ with $\alpha \sigma=\alpha(\sigma \zeta \alpha=\zeta \alpha)$. But $\sigma \zeta \in E_{0}(H)$ $\left[\zeta \sigma \in E_{0}(H)\right]$. Therefore, $a \zeta \in X(\zeta \alpha \in X)$. Hence, $X$ is also a right (left) ideal in the ring $E$.

In preparation for our characterization of the rings $E_{R}(H)$, we establish a few more properties of the finite $R$-endomorphisms of $H$. First, we determine the Jacobson radical of $E_{0}(H)$.

(3.9) Theorem. Let $E$ be a subring of $E_{R}(H)$ which contains $E_{0}(H)$. Then

$$
J\left[E_{0}(H)\right]^{n}=J(E)^{n} \cap E_{0}(H)=\Lambda\left(p^{n} H\right) \cap E_{0}(H)
$$

for every positive integer $n$. Moreover, considered as a ring, $E_{0}(H)$ is simple (not a zero-ring) modulo its Jacobson radical.

Proof. $J\left[E_{0}(H)\right]=J(E) \cap E_{0}(H)$, since $E_{0}(H)$ is a two-sided ideal of $E$. Hence, $J\left[E_{0}(H)\right]^{n} \subseteq J(E)^{n} \cap E_{0}(H)$. And it follows from (2.1) that $J(E)^{n} \cap E_{0}(H)$ $\subseteq \Lambda\left(p^{n} H\right) \cap E_{0}(H)$.

Next let $\zeta \in \Lambda\left(p^{n} H\right) \cap E_{0}(H)$. Then (3.2) implies the existence of a decomposition $H=F \oplus K$ of $H$ with $K \subseteq K(\zeta), H \zeta \subseteq F$ and $F$ of finite rank. Let $\pi$ be the projection of $H$ onto $F$ along $K$. Then $\pi \in E_{0}(H)$ and $\zeta \in \pi E_{\pi}=\pi E_{0}(H)_{\pi}$. But $\pi E_{0}(H)_{\pi}$ is essentially $E_{R}(F)$. Hence, $\zeta \in \Lambda_{E(F)}\left(p^{n} F\right)$. Since $F$ is free of finite rank, it is complete in its $p$-adic topology. Therefore, by (2.3), we have $\Lambda_{E(F)}(p F)=J\left[E_{R}(F)\right]$, which implies $\Lambda_{E(F)}\left(p^{n} F\right)=J\left[E_{R}(F)\right]^{n}$. Since $\pi$ is an idempotent in $E_{0}(H)$, we obtain

$$
J\left[E_{R}(F)\right]^{n}=\left[J\left(\pi E_{0}(H) \pi\right)\right]^{n}=\left[\pi E_{0}(H) \pi \cap J\left(E_{0}(H)\right)\right]^{n} \subseteq J\left[E_{0}(H)\right]^{n} .
$$

Hence, $\zeta \in J\left[E_{0}(H)\right]^{n}$, which shows that $\Lambda\left(p^{n} H\right) \cap E_{0}(H) \subseteq J\left[E_{0}(H)\right]^{n}$. This establishes the desired equality of the three ideals.

Finally, to prove the statement about $E_{0}(H) / J\left[E_{0}(H)\right]$, note that $p H$ is a fully invariant submodule of $H$. Therefore, every $a \in E_{0}(H)$ induces a finite $R / p R$-endomorphism $\alpha^{*}$ in $H / p H$. It is easy to verify that the map $a \rightarrow a^{*}$ constitutes an epimorphism from $E_{0}(H)$ onto $E_{0}(H / p H)$ with kernel $\Lambda(p H) \cap E_{0}(H)=$ $J\left[E_{0}(H)\right]$ (see [5, Lemma 7.4]). The well-known fact that $E_{0}(H / p H)$ is a simple ring $[1$, Structure Theorem, p. 75$]$ completes the proof.

If $H$ is complete, then $E_{0}(H)$ is contained in every nonradical two-sided ideal of $E_{R}(H)$ [4, Theorem 7.5]. This is no longer true for arbitrary $H$. For if we take $H$ to be free of infinite rank, then (2.4) tells us that $\Lambda(p H)$ is a nonradical ideal; but clearly $E_{0}(H) \nsubseteq \Lambda(p H)$.

However, we can establish 
(3.10) Proposition. Let $E$ be a subring of $E_{R}(H)$ wbich contains $E_{0}(H)$. Let $Z$ be a two-sided ideal of $E$. If $Z \cap E_{0}(H)$ is nonradical then $E_{0}(H) \subseteq Z$.

Proof. From (3.9) we derive that $Z \cap E_{0}(H)+J\left[E_{0}(H)\right]=E_{0}(H)$. Suppose that $\sigma^{2}=\sigma \in E_{0}(H)$. Then $\sigma=z+j$ with $z \in Z$ and $j \in J\left[E_{0}(H)\right]$. Also $\sigma=$ $\sigma z \sigma+\sigma j \sigma$. But $\sigma j \sigma \in J(E) \cap \sigma E \sigma=J(\sigma E \sigma)$. Therefore, $\sigma-\sigma j \sigma$ is a unit in $\sigma E \sigma$. Consequently,

$$
\sigma=(\sigma-\sigma j \sigma)(\sigma-\sigma j \sigma)^{-1}=\sigma z \sigma(\sigma-\sigma j \sigma)^{-1} \in Z .
$$

Accordingly, $Z$ contains all idempotents of $E_{0}(H)$. If now $\alpha$ is any element of $E_{0}(H)$, then (3.2) ensures that there exists an idempotent $\tau \in E_{0}(H)$ with $a=\tau a$. Therefore, $a \in Z$, since $Z$ is an ideal containing $\tau$. Hence $E_{0}(H) \subseteq Z$, and the proof is complete.

(3.11) Proposition. Let $E$ be a subring of $E_{R}(H)$ which contains $E_{0}(H)$. Let $Y$ be a minimal nonradical right [left] ideal of $E$. Then there exists a nonleft [right]-zero-divisor $\pi$ in $Y$ with $\pi \in Y \cap J(E)$ and $Y \cap J(E)=\pi Y[=Y \pi]$.

Proof. First suppose that $Y$ is a minimal nonradical right ideal of $E$. By (3.6) there exist $b \in H$ and $B \subseteq H$ such that $H=\{b\} \oplus B$ and $Y=P_{E}(B)$. Denote by $\pi$ the $R$-endomorphism of $H$ in $Y$ defined by $b \pi=p b$ and $B \pi=0$. Let $\phi \epsilon$ $Y \cap J(E)$. Then it follows from (3.7) and (3.9) that $\phi \in \Lambda_{E}(p H)$. Therefore, there exists $y \in H$ such that $b \phi=p y$. If now $\beta$ is the $R$-endomorphism defined by $b \beta=y$ and $B \beta=0$, then $\beta \in Y$ and $b \pi \beta=b \phi$, so that $\phi=\pi \beta$. Accordingly, $Y \cap J(E) \subseteq \pi Y$. It is a consequence of (3.9) that $\pi \in J(E)$, and therefore the opposite inclusion also holds. Thus, $Y \cap J(E)=\pi Y$.

Next we show that $\pi$ is not a left-zero-divisor in $Y$. Suppose that $\alpha \in Y$ and $\pi \alpha=0$. Then $0=b \pi \alpha=p b \alpha$ implies that $b \alpha=0$, since $H$ is torsion-free. Together with $B a=0$ this yields $\alpha=0$, which establishes the proposition in the in the right ideal case.

Now suppose that $X$ is a minimal nonradical left ideal of $E$. By (3.6), $H=$ $\{g\} \oplus A$ for some $g \in H$ and $A \subseteq H$ with $X=\Lambda_{E}(\{g\})$. Let $\omega \in E$ be defined by $g \omega=p g$ and $A \omega=0$. Then $\omega \in X$, and it follows from (3.9) that $\omega \in J(E)$. Therefore, $X \omega \subseteq X \cap J(E)$. To prove the opposite inclusion, pick any $\gamma \in X \cap J(E)$. By (3.7) and (3.9), $y \in \Lambda_{E}(p H)$. Moreover, by (3.2), we can write $\dot{H}=\{x\} \oplus K(\gamma)$ for suitable $x \in H$. Then $x y=p r g$ for some $r \in R$. Note that fox every $r \in R$ there is one only one $r^{\prime} \in R$ such that $p r=r^{\prime} p$. So let $\tau \in x$ be defined by $x \tau=r^{\prime} g$ and $K(\gamma) \tau=0$. Then

$$
x y=p r g=r^{\prime} p g=r^{\prime} g \omega=x \tau \omega, \quad K(\gamma) y=0=K(\gamma) \tau \omega .
$$

Consequently, $\gamma=\tau \omega$, so that $X \cap J(E) \subseteq X \omega$. Hence $X \omega=X \cap J(E)$.

To complete the proof we must show that $\omega$ is not a right-zero-divis or in $X$. So suppose that $\lambda \in X$ and $\lambda \omega=0$. If $H \lambda$ were different from zero, then $H \lambda$ 
would equal some $p^{n}\{g\}$, so that

$$
0=H \lambda \omega=p^{n}\{g\} \omega=p^{n+1}\{g\}
$$

which is a contradiction. Accordingly, $H \lambda=0$. Hence $\lambda=0$, as required.

(3.12) Lemma. Let $H=A \oplus B$ with $A \neq 0 \neq B$. Suppose that $M$ is a minimal nonradical right ideal of $E_{R}(H)$. Then

$$
M=[M \cap \Lambda(A)] \oplus[M \cap \Lambda(B)]=\Lambda_{M}(A) \oplus \Lambda_{M}(B),
$$

where $\Lambda_{M}(A) \neq 0$ and $\Lambda_{M}(B) \neq 0$.

Proof. By (3.6) there exist $b \in H$ and $G \subseteq H$ such that $H=\{b\} \oplus G$ and $M=P(G)$. The lemma follows from

$$
\operatorname{Hom}_{R}(\{b\}, A \oplus B)=\operatorname{Hom}_{R}(\{b\}, A) \oplus \operatorname{Hom}_{R}(\{b\}, B) .
$$

(3.13) Lemma. Let $E$ be a subring of $E_{R}(H)$ which contains a minimal nonradical right ideal $M$ of $E_{R}(H)$. Suppose further that $L$ is a left ideal of $E$. Then $H L$ is a submodule of $H$.

Proof. By (3.6), $M=P(G)$ where $H=\{b\} \oplus G$. Let $a=x \lambda_{1}$ and $b=y \lambda_{2}$ with $\lambda_{1}, \lambda_{2} \in L$. We have to show that there exists $\lambda_{3} \in L$ and $c \in H$ such that $a+b=c \lambda_{3}$. Define the $R$-endomorphisms $a$ and $\beta$ of $H$ by $b a=x, G \alpha=0=G \beta$ and $b \beta=y$. Then $\alpha, \beta \in M \subseteq E$ and

$$
a+b=x \lambda_{1}+x \lambda_{2}=b a \lambda_{1}+b \beta \lambda_{2}=b\left(a \lambda_{1}+\beta \lambda_{2}\right) .
$$

Hence $c=b$ and $\lambda_{3}=a \lambda_{1}+\beta \lambda_{2}$. Furthermore, if $z \lambda \in H L$ and $r \in R$ then clearly $r(z \lambda)=(r z) \lambda \in H L$.

(3.14) Lemma. Let $E$ be a subring of $E_{R}(H)$ which contains a minimal nonradical right ideal $M$ of $E_{R}(H)$. Then $H \Lambda_{E}(S)=S$ for every submodule $S$ of $H$.

Proof. By (3.6), $H=\{b\} \oplus G$ with $M=P(G)$. Clearly $H \Lambda_{E}(S) \subseteq S$. Conversely, if $s \in S$ then there exists $a \in M \subseteq E$ such that $s=b a$. Then $a \in \Lambda_{E}(S)$ shows that $S \subseteq H \Lambda_{E}(S)$, which finishes the proof.

We shall say that a left ideal $L$ of a ring $K$ has a complement in $K$ if there is a left ideal $Q$ in $K$ such that $K$ is the direct sum of $L$ and $Q$.

(3.15) Proposition. Let $E$ be a subring of $E_{R}(H)$ which contains $E_{0}(H)$. Let $M$ be a minimal nonradical right ideal of $E$. If $L$ is a left ideal of the ring $M$ such that $\bigcap_{i=1}^{\infty}\left[\Re_{E}(L)\right]^{i}$ is radical then $L$ cannot bave a nonzero complement in $M$. 
Proof. By (3.6), $H=\{b\} \oplus G$ with $M=P(G)$. Clearly $H M=H$. Suppose that $M=L_{1} \oplus L_{2}$, where $L_{1}$ and $L_{2}$ are left ideals of $M$. Then $H=H M=$ $H\left(L_{1} \oplus L_{2}\right) \subseteq H L_{1}+H L_{2}$. Hence $H=H L_{1}+H L_{2}$. By (3.13), $H L_{1}$ and $H L_{2}$ are submodules of $H$. Their sum must be direct, because $b \lambda_{1}=b \lambda_{2}$ with $\lambda_{1} \in L_{1}$ and $\lambda_{2} \in L_{2}$ implies $\lambda_{1}=\lambda_{2}=0$. By (1.6), $R_{E}\left(L_{1}\right)=P_{E}\left(H L_{1}\right)$. If now $H L_{2}$ were different from zero, then, by (1.1), it would have nonzero cyclic direct summands of $H$; and consequently $E$ would contain minimal idempotents annihilating $H L_{1}$. Therefore, if $\bigcap_{i=1}^{\infty} R_{E}\left(L_{1}\right)^{i}$ is radical, then $H L_{2}=0$, so that $L_{2}=0$. This completes the proof.

(3.16) Proposition. Let $E$ be a subring of $E_{R}(H)$ which contains $E_{0}(H)$. Suppose that $L$ is a left ideal and $M$ a right ideal of $E$. If $L \neq 0$ and $M \neq 0$, then $L \cap M \neq 0$.

Proof. Let $0 \neq \lambda \in L$ and $0 \neq a \in M$. Then there exist $x, y \in H$ such that $x \alpha \neq 0 \neq y \lambda$. Let $b(x \alpha)=n$. By (3.1), there exists $\phi \in E$ with $(x \alpha) \phi=p^{n} y$.

Then

$$
0 \neq p^{n}(y \lambda)=\left(p^{n} y\right) \lambda=((x \alpha) \phi) \lambda=x(\alpha \phi \lambda)
$$

shows that $a \phi \lambda \neq 0$. But $a \phi \lambda \in L r \cdot M$, and so all is proved.

After collecting three further preparatory lemmas, we are ready for the ringtheoretical characterization of the rings between $E_{0}(H)$ and $E_{R}(H)$.

Let $\sigma$ be a minimal idempotent of $E_{R}(H)$. Then by (3.3), $H \sigma$ has rank one. Consequently, $H \sigma$ is complete in its $p$-adic topology. Since $\sigma E_{R}(H) \sigma \cong E_{R}(H \sigma)$, we therefore obtain from (2.3)

(3.17) Proposition. Let $E$ be a subring of $E_{R}(H)$ which contains $E_{0}(H)$. Let $\sigma$ be a minimal idempotent of $E$. Then the ring $\sigma E \sigma$ is Hausdorff and complete in its J-adic topology.

(3.18) Lemma. Let $E$ be an abstract ring with $\bigcap_{n=1}^{\infty} J(E)^{n}=0$. Let $K$ be a minimal nonradical right ideal of $E$. Suppose that there exists a nonnilpotent element $p$ in $K \cap J(E)$ such that $K \cap J(E)=p K$. Then $K=e E$, where $e^{2}=e \in E$. If, in addition, $p$ is not a left-zero-divisor in $K$, and if there exists an element $q$ in $E e \cap J(E)$ such that $E e \cap J(E)=E e q$, then eEe is a discrete valuation ring with $J(e E e)=p e E e=e E e p$.

Proof. If $k^{2} \in K \cap J(E)$ for all $k \in K$, then $K+J(E) / J(E)$ would be nil and consqeuently, $K \subseteq J(E)$. Therefore, we can find an element $k \in K$ such that $k^{2} \notin J(E)$. The minimality of $K$ implies $K=k K=k E=k^{2} E$.

Now consider $S=\{\phi \in K \mid k \phi \in J(E)\}$. Then $S$ is a right ideal of $E$. If $S$ were not contained in $J(E)$, then $S=K$, by the minimality of $K$. This, however, would imply $k^{2} \in J(E)$ and violate our choice of $k$. Therefore, if for some $\alpha \in K$ 
we have $k a \in J(E)$, then $\alpha \in J(E)$. Among other things, this implies $k[K \cap J(E)]^{n}$ $=[K \cap J(E)]^{n}$ for all $n \geq 1$. Note that our hypothesis implies immediately that $[K \cap J(E)]^{n}=p^{n} K$ for all $n \geq 1$. Also, we obtain from $\bigcap_{n=1}^{\infty} J(E)^{n}=0$ that every nonzero element $\alpha$ of $K$ has a representation of the from $\alpha=p^{m} \alpha^{\prime}$ with $\alpha^{\prime} \notin$ $J(E)$ and $m \geq 0$.

Next we consider $\Re_{K}(k)$, the right annihilator of $k$ in $K$. We wish to show that $R_{K}(k)=0$. So let $0 \neq \alpha \in \mathscr{R}_{K}(k)$. Then $\alpha \in S$, hence $a \in J(E)$. Write $a=$ $p^{m} \alpha^{\prime}$ with $\alpha^{\prime} \notin J(E)$ and $m \geq 1$. Then $\alpha^{\prime} \in \mathcal{R}_{K}\left(k p^{m}\right)$. If the right ideal $R_{K}\left(k p^{m}\right)$ were not radical, then it would be all of $K$, so that

$$
0=k p^{m} K=k[K \cap J(E)]^{m}=[K \cap J(E)]^{m}
$$

would imply $p^{m}=0$, which is impossible. Hence $R_{K}\left(k p^{m}\right) \subseteq J(E)$, so that $\alpha^{\prime} \epsilon$ $J(E)$, which is likewise a contradiction. It follows that $R_{K}(k)=0$.

The wanted idempotent generator for $K$ is now easily found. Since $k K=K$, there exists a nonzero element $e \in K$ such that $k e=k$. Then $e^{2}-e \in R_{K}(k)$, hence $e=e^{2}$. Clearly $e \notin J(E)$. Since $K$ is minimal nonradical, we obtain $K=$ $e E$.

Now $e E e$ is a ring with identity $e$. By (3.5), eEe/J(eEe) is a division ring. Moreover,

$$
J(e E e)=e E e \cap J(E)=e E \cap E e \cap J(E)
$$

is true in any ring $E$. Next assume that $E e \cap J(E)=E e q$. Then

$$
[e E \cap J(E)] \cap E e=p e E \cap E e=p e E e
$$

and

$$
[E e \cap J(E)] \cap e E=E e q \cap e E=e E e q .
$$

Hence, $J(e E e)=p e E e=e E e q$. Therefore, we can find $\beta \in e E e$ such that $p e=$ $\beta q$. Suppose that $\beta \in J(e E e)$. Then $\beta=p y$ with $\gamma \in e E e$. Assuming that $p$ is not a left-zero-divisor in $e E$, the equation $p e=p \gamma q$ implies that $e=\gamma q$. But $\gamma q \in J(E)$. This contradicts the fact that the Jacobson radical contains no nonzero idempotents. It follows that $\beta \notin J(e E e)$. Since $e E e$ is a local ring, $\beta$ must be a unit in $e E e$. Therefore, $e E e p=e E e \beta q=e E e q$. Thus, $J(e E e)=$ $p e E e=e E e p$. We know that $p$ is not nilpotent. Finally, observe that

$$
\bigcap_{i=1}^{\infty} J(e E e)^{i}=\bigcap_{i=1}^{\infty}[e E e \cap J(E)]^{i} \subseteq \bigcap_{i=1}^{\infty} J(E)^{i}=0 .
$$

Therefore, we see from our definition in $\$ 1$ that $e E e$ is a discrete valuation ring, and now the proof is complete.

Next we show how we can recapture $H$ from its endomorphism ring. Note that $\sigma E$ is a minimal nonradical right ideal of $E$. 
(3.19) Proposition. Let $E$ be a subring of $E_{R}(H)$ which contains $E_{0}(H)$. Suppose that $\sigma$ is a minimal idempotent of $E$. Then the $R$-module $H$ is isomorphic with the $\sigma E \sigma-m o d u l e ~ \sigma E$.

Proof. By (3.3), we have $H=H \sigma \oplus K(\sigma)$, where $H \sigma=R x$ has rank one. To every $r \in R$ there is one and only one $R$-endomorphism $r^{\xi}=\sigma r^{\xi} \sigma$ of $H$ with the properties $x r^{\xi}=r x$ and $K(\sigma) r^{\xi}=0$. And to every element $b \in H$ there exists one and only one $R$-endomorphism $b^{\xi}=\sigma b^{\xi}$ of $H$ with the properties $x b^{\xi}=b$ and $K(\sigma) b^{\xi}=0$. It is easy to check that $\xi$ effects the required isomorphism.

(3.20) Theorem. Let $E$ be an abstract ring with Jacobson radical $J(E)$. Then there exists a complete discrete valuation ring $R$ and a reduced torsionfree $R$-module $H$ such that $E$ is isomorpbic to a subring of $E_{R}(H)$ containing $E_{0}(H)$ if and only if the following conditions bold:

(1) $\bigcap_{n=1}^{\infty} J(E)^{n}=0$.

(2) E contains minimal nonradical right ideals.

(3) If $M$ is a minimal nonradical right [left] ideal of $E$, then there exists a non-left [right]-zero-divisor in $M$ such that $p \in M \cap J(E)$ and $M \cap J(E)=p M$ [resp. $M \cap J(E)=M p]$.

(4) Let $M$ be a minimal nonradical right ideal of $E$. If $L$ is a left ideal of the ring $M$ such that its right annibilator in $E$ satisfies $\bigcap_{i=1}^{\infty} R_{E}(L)^{i}=0$, then $L$ cannot bave a nonzero complement in $M$.

(5) Let $L$ be a left ideal and $K$ a right ideal of $E$. If $L \neq 0$ and $K \neq 0$, then $L \cap K \neq 0$.

(6) If $e$ is a minimal idempotent in $E$, then the ring $e E e$ is complete in its J-adic topology.

Remark. We note that in (3) we require the validity of both statements regarding minimal nonradical right and left ideals.

Proof. The necessity of (1) follows from (2.2), and the necessity of (2) follows from (1.1) and (3.6). Condition (3) is necessary by virtue of (3.11), and condition (4) by virtue of (3.15). Finally (3.16) shows the necessity of (5), while (6) is necessary because of (3.17).

Now assume that $E$ satisfies (1)-(6). By (2) there exists a minimal nonradical right ideal $H$ of $E$. It follows from (1) and (3) together with (3.18) that $H=e E$, where $e^{2}=e \in E$. Then by (3.5), $E e$ is a minimal nonradical left ideal. Therefore (3) together with (3.18) implies that $R=e E e$ is a discrete valuation ring with $p=e p e$ as a two-sided generator of its Jacobson radical. Moreover, by (6), $R$ is complete since $e$ must necessarily be a minimal idempotent. Note that $H$ is a left $R$-module. It follows from (3) that 0 is the only element of $H$ 
which is annihilated on the left by $p$. In addition, we obtain from (1) that $\bigcap_{n=1}^{\infty} p^{n} H=0$. Thus, $H$ is torsion-free and reduced as an $R$-module. The right annihilator $R(H)$ of $H$ is a two-sided ideal of $E$. Since $e$ is a left identity on $H$, we must have $R(H) \cap H=0$. Therefore (5) implies that $R(H)=0$, so that $H$ is a faithful right $E$-module. This enables us to identify $E$ with a subring of the $R$-endomorphism ring $E_{R}(H)$ of the left $R$-module $H$. In the following we shall use the operators $\Lambda$ and $P$ as defined in $\S 1$.

We wish to show that $E$ contains all finite $R$-endomorphisms of $H$. Because of (1.2) and (3.2) it is only necessary to show that to each decomposition $H=$ $\{b\} \oplus G$ and each $x \in H$ there exists an $R$-endomorphism in $E$ which maps $b$ onto $x$ and annihilates $G$. To this end we first observe that the minimal nonradical right ideal $e E$ of $E$ is actually a minimal nonradical right ideal in the ring $E_{R}(H)$. This is an immediate consequence of (3.6), because we have $H=e E=$ $e E e \oplus e E(1-e)$ and $e E e=R$, where $e E(1-e)$ is the set of elements $y-y e$ with $y \in e E$, so that $e E$ is exactly the set of all $R$-endomorphisms of $H=$ $\{e\} \oplus H(1-e)$ which annihilate the direct summand $H(1-e)$ of corank 1 . Therefore, it follows from (3.12) that $M=\Lambda_{M}(\{b\}) \oplus \Lambda_{M}(G)$ and $\Lambda_{M}(\{b\}) \neq 0 \neq \Lambda_{M}(G)$. This means that $\Lambda_{M}(\{b\})$ and $\Lambda_{M}(G)$ are complementary left ideals of the ring $M$. Hence by (4), the right annihilator $Q=R_{E}\left[\left(\Lambda_{M}(G)\right]\right.$ satisfies $\bigcap_{i=1}^{\infty} Q^{i} \neq 0$. By $(3,14)$, we have $H \Lambda_{M}(G)=G$. Therefore, we obtain $Q=P_{E}(G)$ from (1.6). Now $H Q \subseteq p H$, for otherwise $H\left(\bigcap_{i=1}^{\infty} Q^{i}\right) \subseteq \bigcap_{i=1}^{\infty} p^{i} H=0$ would imply $\bigcap_{i=1}^{\infty} Q^{i}$ $=0$, and this, as we have seen, is impossible. But $p H=H \cap J(E)$. Therefore, $H Q=b Q$ is a nonradical right ideal contained in $H$. Then the minimality of $H$ implies $b Q=H$ and the theorem is proved.

We conclude this section with the following structure theorem which establishes the uniqueness of the module $H$ in (3.20). If $R$ is the set of rational numbers which can be written as fractions prime to a prime $p$, then there exist nonisomorphic reduced torsion-free $R$-modules having isomorphic endomorphism rings. For complete $R$, however, the world is much better:

(3.21) Theorem. Let $H, H^{\prime}$ be reduced torsion-free modules over complete discrete valuation rings $R, R^{\prime}$ respectively. Let $E, E^{\prime}$ be subrings of $E_{R}(H)$, $E_{R^{\prime}}\left(H^{\prime}\right)$ respectively which contain $E_{0}(H), E_{0}\left(H^{\prime}\right)$ respectively. If now $E$ is isomorphic to $E^{\prime}$ then $H$ is isomorphic to $H^{\prime}$.

Proof. Under a ring isomorphism minimal idempotents are mapped onto minimal idempotents. Therefore, the assertion follows from (3.19).

4. The finite topology. In this section we shall characterize the rings $E_{R}(H)$. In view of (3.20), all we have to do is this: if $E$ is a subring of $E_{R}(H)$ which contains $E_{0}(H)$, then find a property of $E$ which is characteristic for the particular 
case $E=E_{R}(H)$. For complete modules $H$ we could solve this problem by requiring the existence of an identity element and a certain condition on the left annihilators in $E$ (see [4, Theorem 9.2]). Unfortunately, this method does not work if we drop the hypothesis that $H$ be complete. The only solution we can offer is a topological one: completeness of $E$ in its finite topology.

Definition. Let $E$ be a subring of $E_{R}(H)$. Then the family $P_{E}(F)=$ $\{\alpha \in E \mid F \alpha=0\}$, where $F$ runs over all finite subsets of $H$, constitutes a neighborhood basis at 0 for a topology on $E$ which we call the finite topology.

Clearly the finite topology on $E$ is Hausdorff. And the simple fact $P_{E}(F)=$ $E \cap P(F)$ shows that the finite topology on $E$ is the same as the relative topology induced by the finite topology of $E_{R}(H)$. For our purpose we need an abstract definition of the finite topology on $E$, without referring to the underlying module.

Again we assume that $E$ is a subring of $E_{R}(H)$ which contains $E_{0}(H)$. Let $F$ be a finite subset of $H$. Then the purification

$$
\{F\}^{*}=\{b \in H \mid r b \in\{F\} \text { for some } r \neq 0 \text { in } R\}
$$

has finite rank and is therefore, by (1.3), a direct summand of $H$. It readily follows that $P_{E}\left(\{F\}^{*}\right)=P_{E}(F)$. Now let $\sigma$ be a projection of $H$ onto $\{F\}^{*}$. Then $\sigma \in E_{0}(H) \subseteq E$. Moreover, applying (1.6), $P_{E}\left(\{F\}^{*}\right)=P_{E}(H \sigma)=R_{E}(\sigma)$. Hence, every $P_{E}(F)$ is the right annihilator in $E$ of a finite subset of $E_{0}(H)$. Conversely, let $T$ be a finite subset of $E_{0}(H)$. Then the submodule $\{H T\}$ generated by $H T$ has finite rank, hence is free by (1.2), and can therefore be generated by a finite subset $F$. of $H$. It follows from (1.6) that $R_{E}(T)=P_{E}(H T)=P_{E}(\{H T\})=$ $P_{E}(F)$. Accordingly, we obtain

(4.1) Proposition. Let $E$ be a subring of $E_{R}(H)$ which contains $E_{0}(H)$. Then the finite topology on $E$ coincides with the topology on $E$ obtained by taking the right annibilators in $E$ of the finite subsets of $E_{0}(H)$ as a neighborbood system of 0 .

Remark. The reader will observe that the finite topology on $E$ can also be defined by choosing the right annihilators of the finite idempotents of $E$ as a neighborhood basis at 0 . Pierce did that in [6] for the endomorphism rings of p-groups without elements of infinite height.

(4.1) and (3.7) suggest the following abstract definition of the finite topology on an arbitrary ring. If a ring is without minimal nonradical right ideals, then we define the sum of its minimal nonradical right ideals to be the zero ideal.

Definition. Let $E$ be an abstract ring and $E_{0}$ the sum of all its minimal nonradical right ideals. Let $\phi\left(E_{0}\right)$ be the family of all finite subsets of $E_{0}$. Then the family $\left\{R_{E}(S) \mid S \in \Phi\left(E_{0}\right)\right\}$ constitutes a neighborhood basis at 0 for a 
topology on $E$ which we call the finite topology.

(4.2) Lemma. $E_{R}(H)$ is complete in its finite topology [3, Lemma 3.3, p. 252].

(4.3) Lemma. $E_{0}(H)$ is dense in $E_{R}(H)$ in the finite topology.

Proof. Let $\alpha \in E_{R}(H)$ and let $S$ be a finite subset of $H$. We must show that $E_{0}(H) \cap[\alpha+P(S)]$ is not empty. By (1.5) we can embed $S$ into a direct summand $F$ of finite rank. Let $\sigma$ be a projection of $H$ onto $F$. Then $1-\sigma \in P(S)$ and, therefore, $(1-\sigma) \alpha \in P(S)$, since $P(S)$ is a right ideal. But $\sigma \alpha=\alpha-(1-\sigma) \alpha \epsilon$ $E_{0}(H)$.

(4.4) Corollary. Let $E$ be a subring of $E_{R}(H)$ which contains $E_{0}(H)$. Suppose that $E$ is complete in its finite topology. Then $E=E_{R}(H)$.

By combining (3.20) with (4.2) and (4.4) we arrive at the following ringtheoretical characterization of $E_{R}(H)$.

(4.5) Theorem. Let $E$ be an arbitrary ring. Then there exists a complete discrete valuation ring $R$ and a reduced torsion-free $R$-module $H$ such that $E$ is isomorpbic to the ring of all $R$-endomorphisms of $H$ if and only if $E$ satisfies conditions (1)-(6) of (3.20) and is complete in its finite topology.

5. The J-finite topology. The subject of this final section will be a characterization of $E_{R}(H)$ for complete $H$.

Let $E$ be a subring of $E_{R}(H)$. If $F, G$ are subsets of $H$, then define

$$
\Lambda_{E}(F, G)=\{\alpha \in E \mid F \alpha \subseteq G\} .
$$

If $G$ is fully invariant, then $\Lambda_{E}(F, G)$ is a right ideal of $E$.

Definition. Let $\Phi(H)$ be the family of all finite subsets of $H$. Then the family $\left\{\Lambda_{E}\left(F, p^{i} H\right) \mid F \in \Phi(H), i=0,1,2, \ldots\right\}$ constitutes a neighborhood basis at 0 for a topology on $E$ which we call the $J$-finite topology.

We will use the notation $\left\{\xi_{i} \mid i \in D\right\}$, where $D$ is a directed set, to denote a net in $E$. The $J$-finite topology on $E$ is the relative topology induced by the $J$-finite topology on $E_{R}(H)$. It is Hausdorff since $\bigcap_{i=1}^{\infty} p^{i} H=0$. Moreover, endowing $H$ with its $p$-adic topology, we see that the $J$-finite topology is that of pointwise convergence on $H$. Therefore, a net $\left\{\xi_{i} \mid i \in D\right\}$ in $E_{R}(H)$ converges to $\xi$ if and only if $\left\{x \xi_{i} \mid i \in D\right\}$ converges to $x \xi$ for each $x \in H$.

(5.1) Lemma. $E_{R}(H)$ is complete in its J-finite topology if and only if $H$ is complete in its p-adic topology.

Proof. Assume that $E_{R}(H)$ is complete in its $J$-finite topology. Let $\left\{b_{i}\right\}$ be a Cauchy sequence in $H$. We know that $H$ has cyclic direct summands. So 
write $H=\{b\} \oplus G$ and define $R$-endomorphisms $a_{i}$ by $b a_{i}=b_{i}$ and $G a_{i}=0$. Then $\left\{\alpha_{i}\right\}$ is a Cauchy sequence in the $J$-finite topology of $E_{R}(H)$. Let $\alpha=$ $\lim \alpha_{i}$. Sequential convergence in $E_{R}(H)$ is equivalent to pointwise convergence. Therefore, $\lim b_{i}=\lim \left(b \alpha_{i}\right)=b \alpha$.

Conversely, assume that $H$ is complete. Let $\left\{\xi_{i} \mid i \in D\right\}$ be a Cauchy net in $E_{R}(H)$ and pick $g \in H$. Then $\left\{g \xi_{i} \mid i \in D\right\}$ is a Cauchy net in the p-adic topology of $H$. Let $x_{g}=\lim \left(g \xi_{i}\right)$. Define $g \xi=x_{g} \in H$. It is easy to verify that $\xi$ is a well-defined $R$-endomorphism of $H$. Hence $\left\{\xi_{i} \mid i \in D\right\}$ converges pointwise to $\xi$. Therefore, $E_{R}(H)$ is complete in its $J$-finite topology.

(5.2) Lemma. $E_{0}(H)$ is dense in $E_{R}(H)$ in the J-finite topology.

Proof. The J-finite topology of $E_{P}(H)$ is coarser than the finite topology: $P(S)=\bigcap_{i=1}^{\infty} \Lambda\left(S, p^{i} H\right)$. Therefore, the assertion follows from (4.3).

(5.3) Corollary. Let $E$ be a subring of $E_{R}(H)$ which contains $E_{0}(H)$. Suppose that $E$ is complete in its J-finite topology. Then $E=E_{R}(H)$, and $H$ is complete in its p-adic topology.

Let us now show how the $J$-finite topology can be defined on $E_{R}(H)$ without referring to $H$. To this end, let $E$ be again a subring of $E_{R}(H)$ which contains $E_{0}(H)$. Assume that $S=\left\{s_{1}, \ldots, s_{n}\right\}$ is a finite subset of $H$. Also choose a decomposition $H=\{b\} \oplus G$ of $H$. For $j=1, \ldots, n$ define $R$-endomorphisms $a_{j}$ of $H$ by $b \alpha_{j}=s_{j}$ and $G \alpha_{j}=0$. Let $\Gamma=\left\{a_{j} \mid j=1, \cdots, n\right\}$. Then $\Gamma \subseteq E_{0}(H)$ and $S=H \Gamma$. Hence, $S a \subseteq p^{i} H$ implies $H \Gamma a \subseteq p^{i} H$. Thus

$$
\Lambda_{E}\left(S, p^{i} H\right)=\left\{\alpha \in E \mid \Gamma \alpha \subseteq \Lambda_{E}\left(p^{i} H\right)\right\}
$$

But $\Gamma a \subseteq E_{0}(H)$ for all $\alpha \in E$, since $E_{0}(H)$ is a right ideal. Therefore, we see from (3.9) that

$$
\Lambda_{E}\left(S, p^{i} H\right)=\left\{\alpha \in E \mid \Gamma \alpha \subseteq J(E)^{i}\right\}
$$

Conversely, let $\theta$ be a finite subset of $E_{0}(H)$. Consider $N=\left\{\alpha \in E \mid \theta \alpha \subseteq J(E)^{i}\right\}$. It is an immediate consequence of (3.9) that $N=\Lambda_{E}\left(\{H \theta\}, p^{i} H\right)$. But the submodule $\{H \theta\}$ has finite rank and is therefore free, by (1.2). Hence, it can be generated by a finite subset $F$ of $H$. Now obviously $N=\Lambda_{E}\left(F, p^{i} H\right)$. Accordingly we have proved

(5.4) Proposition. Let $E$ be a subring of $E_{R}(H)$ which contains $E_{0}(H)$. Let $\Phi\left(E_{0}\right)$ be the family of all finite subsets of $E_{0}(H)$. For each $\Gamma \in \Phi\left(E_{0}\right)$ and each integer $i \geq 0$ define $N(\Gamma, i)=\left\{a \in E \mid \Gamma \alpha \subseteq J(E)^{i}\right\}$. Then the J-finite topology on $E$ coincides with the topology obtained by taking the family $\{N(\Gamma, i) \mid$ $\left.\Gamma \in \Phi\left(E_{0}\right), i \geq 0\right\}$ as a neighborbood bas is at zero. 
(5.4) and (3.7) now suggest the following abstract definition of the J-finite topology on an arbitrary ring.

Definition. Let $E$ be an arbitrary ring and $E_{0}$ the sum of all its minimal nonradical right ideals. Let $\Phi\left(E_{0}\right)$ be the family of all finite subsets of $E_{0}$. For each $\Gamma \in \Phi\left(E_{0}\right)$ and each $i \geq 0$ define $N(\Gamma, i)=\left\{\alpha \in E \mid \Gamma \alpha \subseteq J(E)^{i}\right\}$. The family $\left\{N(\Gamma, i) \mid \Gamma \in \Phi\left(E_{0}\right), i \geq 0\right\}$ constitutes a neighborhood basis at 0 for a topology on $E$ which we call the $J$-finite topology.

We now obtain the following characterization of $E_{R}(H)$ for complete $H$ by combining (3.20) with (5.2) and (5.3).

(5.5) Theorem. Let $E$ be an arbitrary ring. Then there exists a complete discrete valuation ring $R$ and a reduced complete torsion-free $R$-module $H$ such that $E$ is isomorphic to the ring of all R-endomorphisms of $H$ if and only if $E$ satisfies conditions (1)-(5) of (3.20) and is complete in its J-finite topology.

Remark. The reader will notice that in (5.5) we dropped condition (6) of (3.20). The purpose of requiring (6) in (3.20) was to guarantee completeness in the $J$ adic topology of the discrete valuation ring $e E e$, where $e E$ was a minimal nonradical right ideal $\left(e^{2}=e\right)$. It is easy to verify that in $(5.5)$ this is a consequence of the completeness of $E$ with respect to its $J$-finite topology.

\section{REFERENCES}

1. N. Jacobson, Structure of rings, Amer. Math. Soc. Colloq. Publ., vol. 37, Amer. Math. Soc., Providence, R. I., 1956. MR 18, 373.

2. I. Kaplansky, Infinite abelian groups, Univ. of Michigan Press, Ann Arbor, Mich., 1954. MR 16, 444 .

3. W. Liebert, Endomorphism rings of abelian p-groups, Studies on Abelian Groups (Sympos., Montpellier, 1967), Springer, Berlin, 1968, pp. 239-258. MR 39 \#4273.

4. Characterization of the endomorphism rings of the divisible torsion modules and reduced complete torsion-free modules over complete discrete valuation rings, Pacific J. Math. 37 (1971), 141-170.

5. R. S. Pierce, Homomorphisms of primary abelian groups, Topics in Abelian Groups (Proc. Sympos., New Mexico State Univ., 1962), Scott, Foresman, Chicago, Ill., 1963. MR $31 \# 1299$.

6. - Endomorphism rings of primary A belian groups, Proc. Colloq. Abelian Groups (Tihany, 1963), A kad. Kiadó, Budapest, 1964, pp. 125-137. MR 30 \#137.

7. K. G. Wolfson, An ideal-theoretic characterization of the ring of all linear transformations, Amer. J. Math. 75 (1953), 358-386. MR 14, 718.

DEPARTMENT OF MATHEMATICS, NEW MEXICO STATE UNIVERSITY, LAS CRUCES, NEW MEXICO 88001 